\title{
Cancro do Esófago: Estratégia de Abordagem Cirúrgica
}

\author{
Esophageal Cancer: Surgical Strategies
}

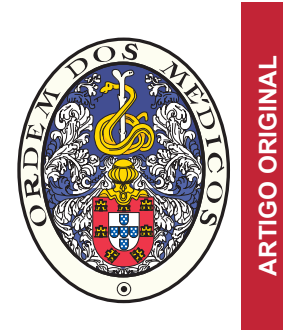

\author{
Paulo COSTA ${ }^{1}$, Rui ESTEVES ${ }^{1}$, Patrícia LAGES ${ }^{1}$, Filipa FERREIRA ${ }^{1}$ \\ Acta Med Port 2014 Sep-Oct;27(5):593-600
}

\section{RESUMO}

Introdução: A ressecção transmediastínica e a ressecção transtorácica têm mortalidade hospitalar $(1,4 \%-14 \%)$ e sobrevivência $( \pm$ $25 \%$ aos cinco anos) semelhantes. A terapêutica neo-adjuvante é opção em estádios avançados. A intenção deste trabalho é apresentar uma série consecutiva de 52 doentes - opção operatória baseada na localização anatómica: tumores infra-carinais e cervicais submetidos a ressecção transmediastínica e restantes ressecções transtorácicas.

Material e Métodos: $O$ estudo incluiu 52 doentes consecutivos, sete mulheres e 45 homens, mediana de idade: 64 anos [46-85]. Localização: cervical - um; montante da carina - 22; jusante da carina -19; cárdia tipo I -10. Histologia: 19 adenocarcinomas, 32 carcinomas pavimento-celulares, um linfoma. Vinte doentes (40\%) - terapêutica neoadjuvante. Abordagem tóraco-abdominal - três, tóraco-abdómino-cervical - 20, transhiatal - 27, toracotomia exploradora - dois. Transposição gástrica 49 (anastomose cervical - 46; torácica - três); esofagocoloplastia cervical - um.

Resultados: Estadiamento patológico: regressão completa - 8; lb - 3; Ila - 9; Ilb - 4; Ila - 11; Illb -2; Illc - 10; IV - 1; linfoma - um; não classificáveis - três. Complicações major: 37\%. Ressecabilidade: 96\% (50/52). Mortalidade: quatro semanas - 6\%; hospitalar - 14\%. Sobrevida mediana 18 meses [3-80]. Curvas de sobrevida (Kaplan-Meier): dois anos - 47\%; cinco anos - $19 \%$.

Discussão: Não tendo sido demonstrada vantagem oncológica para a ressecção transtorácica ou a ressecção transmediastínica, basear a opção operatória na localização do tumor permitiu-nos com segurança e eficácia, planear e executar as ressecções esofágicas desta série.

Conclusão: As curvas de sobrevida foram sobreponíveis para ressecção transtorácica e ressecção transmediastínica e bastante favoráveis numa população com $52 \%$ de estádios pIII/IV. A quimio-radioterapia contribuiu para aumentar a ressecabilidade.

Palavras-chave: Esofagectomia; Laparoscopia; Neoplasias Esofágicas/cirurgia; Toracotomia.

\section{ABSTRACT}

Introduction: Oesophagectomy for cancer is associated to a significant morbidity and mortality. The superiority of transthoracic vs transhiatal is still a matter of controversy. The aim of this paper is to discuss the results of a series of patients submitted to either a transthoracic or a transhiatal according to the anatomic location regarding the carina.

Material and Methods: Retrospective analysis of 52 consecutive patients, with oesophageal carcinoma, 7 female and 45 males, median age 64 [46-85] years. Location: cervical - 1; above carina - 22; below or at carina level - 19; cardia type I-10. 19 adenocarcinoma, 32 squamous cells, 1 lymphoma. Twenty patients $(40 \%)$ - neo-adjuvant therapy. Thoracoabdominal approach - 3, cervico-thoracoabdominal -20 , transhiatal -27 , exploratory thoracotomy -2 .

Gastric pull-up 49 (cervical anastomosis - 46; thoracic - 3); cervical oesofagocoloplasty -1.

Results: Pathologic staging: complete remission - 8; lb - 3; Ila - 9; Ilb - 4; Ila - 11; Illb -2; IIlc - 10; IV - 1; non-stageable - 3. Major complications: $37 \%$. Resectability: $96 \%$ (50/52). Mortality: 4th week - $6 \%$; in-hospital $-14 \%$. Median survival 18 months [3-80]. Survival Curves (Kaplan-Meier): 2 years - 47\%; 5 years - 19\% (transthoracic vs transhiatal $p>0.05$ ).

Discussion: Selection of surgical approach based on the anatomic location of the tumour regarding the carina was safe, the resectability was high and similar when a transthoracic or a transhiatal was planed and carry-on.

Conclusions: In this series of oesophageal cancer patients, in advanced pathologic condition (52\% p Stages III/IV) the overall survival was similar for transthoracic and transhiatal. Neo-adjuvant treatments definitively contributed to enhance resectability.

Keywords: Esophagectomy; Laparoscopy; Esophageal Neoplasms/surgery; Thoracotomy.

\section{INTRODUÇÃO}

À semelhança do que se tem considerado como padrão de actuação oncológica para as outras localizações tumorais no tubo digestivo, também os tumores do esófago são hoje tratados numa perspectiva multimodal em ambiente multidisciplinar. Mas esta evolução do equacionamento e das vias de tratamento está longe de ter trazido consensos suportados por evidência científica.

A abordagem dos tumores do esófago deve ser decidida de acordo com o doente, as características do tumor, a experiência e disponibilidades do grupo multidisciplinar, da equipa cirúrgica e das unidades de suporte de vida. ${ }^{1-4}$
A ressecção do esófago, quando possível, continua a ser a base do tratamento, quer seja quando utilizada isoladamente, quer quando em combinação para melhorar os resultados das outras terapêuticas..$^{5-9}$

A principal questão, que não está ainda definida, centra-se na determinação da equivalência, ou não, da ressecção transtorácica (RTT) em comparação com a abordagem transmediastínica (RTM). Os prós e contras de cada alternativa encontram-se difusamente explicitados nas experiências individuais e revisões sistemáticas publicadas, ${ }^{10-15}$ mas não existe evidência suficiente para propor

1. Clínica Universitária de Cirurgia I. Faculdade de Medicina. Universidade de Lisboa. Hospital de Santa Maria. Centro Hospitalar Lisboa Norte. Lisboa. Portugal. Recebido: 10 de Janeiro de 2014 - Aceite: 23 de Junho de 2014 | Copyright @ Ordem dos Médicos 2014 
recomendações fortemente suportadas.

Recentemente analisámos a nossa experiência com a utilização do tubo gástrico na reconstrução digestiva após esofagectomia por cancro esofágico. ${ }^{16}$ Realizámos preferencialmente a anastomose esófago-gástrica cervical, com excelentes resultados, pelo que a consideramos a primeira escolha na generalidade destes doentes. ${ }^{15-17}$

No tratamento do cancro do esófago, a radioterapia aplicada isoladamente, ou após quimio-sensibilização, como tem sido a nossa preferência, tem demonstrado resultados interessantes, apesar de não ser desprovida de complicações graves e poder necessitar de salvage surgery num número significativo de casos. ${ }^{18-23}$

A intenção deste trabalho foi apresentar os resultados da estratégia multimodal de tratamento do cancro do esófago, tendo sido geralmente decidida a opção da componente operatória do processo com base na localização do tumor.

\section{MATERIAL E MÉTODOS}

Estudo retrospectivo (2006-12), incluindo 52 doentes consecutivos, com carcinoma do esófago. Considerámos sete doentes do sexo feminino e 45 do masculino, com mediana de idade 64 [46-85] anos. O autor PC participou como um dos cirurgiões sénior em todas as cirurgias. Dois doentes foram operados no Hospital CUF Infante Santo (Lisboa) e outros dois no Hospital Beatriz Ângelo (Loures). Localização do tumor: cervical - um; a montante da carina - 22; a jusante da carina -19 e cárdia tipo I - 10. Um dos casos submetido a quimio-radioterapia, com regressão completa, quatro anos antes de ter recidiva, foi considerado para salvage surgery.

Histologia: 19 adenocarcinomas, 32 carcinomas pavimento-celulares e um linfoma (pré-operatoriamente com biopsia de carcinoma indiferenciado).

Vinte doentes foram submetidos a terapêutica neo-adjuvante (19 quimio-radioterapia e um quimioterapia).

Abordagem tóraco-abdominal - três doentes, tóraco-abdómino-cervical - 20 doentes, transhiatal - 27 doentes e toracotomia exploradora - dois doentes.

Transposição gástrica em 49 casos com anastomose cervical em 46 e torácica em três; esofagocoloplastia cervical - um.

- Avaliação diagnóstica e da ressecabilidade: endoscopia com biópsia; eco-endoscopia em casos selecionados; TAC cervico-tóraco-abdómino-pélvica; broncoscopia nos tumores retro-traqueo-brônquicos; PET- scan na suspeita de metastização ganglionar à distância.

- Decisão multidisciplinar oncológica para inclusão dos doentes em programa de terapêutica neo-adjuvante, quimio-radioterapia na generalidade das situações $\mathrm{N}+$, suspeita de aderência a estruturas não ressecáveis ( $T$ IV - b) ou volumosas massas tumorais.

- Avaliação do risco pré-operatório, incluindo parâmetros analíticos sumários (ex.: função hepática, renal e estudo da coagulação), avaliação da função cardíaca (ECG; ecocardiograma) e da função pulmonar (provas de função respiratória; gasimetria arterial e radiografia de tórax) e optimização do status nutricional. ${ }^{24,25}$

- Técnica operatória (RTM e RTT)

RTM - Com o doente em decúbito dorsal, a intervenção começa pelo tempo abdominal e, após ter sido avaliada a possibilidade de prossecução da operação, uma segunda equipa aborda o esófago na região cervical.

Após laparotomia mediana, procedeu-se ao afastamento/suspensão das grelhas costais com afastadores fixados lateralmente, sendo seccionado o ligamento coronário esquerdo para mobilizar o lobo esquerdo do fígado. Para permitir bom acesso ao hiato esofágico foi feita extensa frenotomia - manobra de Pinotti. ${ }^{26} \mathrm{~A}$ ressecção do esófago e esvaziamento ganglionar infra-carinal e abdominal (estações ganglionares $1,2,3,7,9,11,16,19,20,108,110$, $111,112),{ }^{27}$ foram feitos pelo abdómen com bom acesso e controlo da dissecção (Fig. 1). A dissecção do mediastino foi realizada com iluminação adequada, por fitas auto-adesivas contendo fibras ópticas ligadas a gerador de luz, ou, nos últimos casos, recorrendo ao sistema de videoscopia que assegura excelente iluminação e ainda uma visão ampliada das estruturas, praticamente até ao opérculo torácico. Na selagem de vasos utilizámos preferencialmente aparelhos que permitem a secção dos vasos após selagem. Na última metade da série, procurámos laquear electivamente o canal torácico.

A cervicotomia esquerda foi efectuada com a face volta-
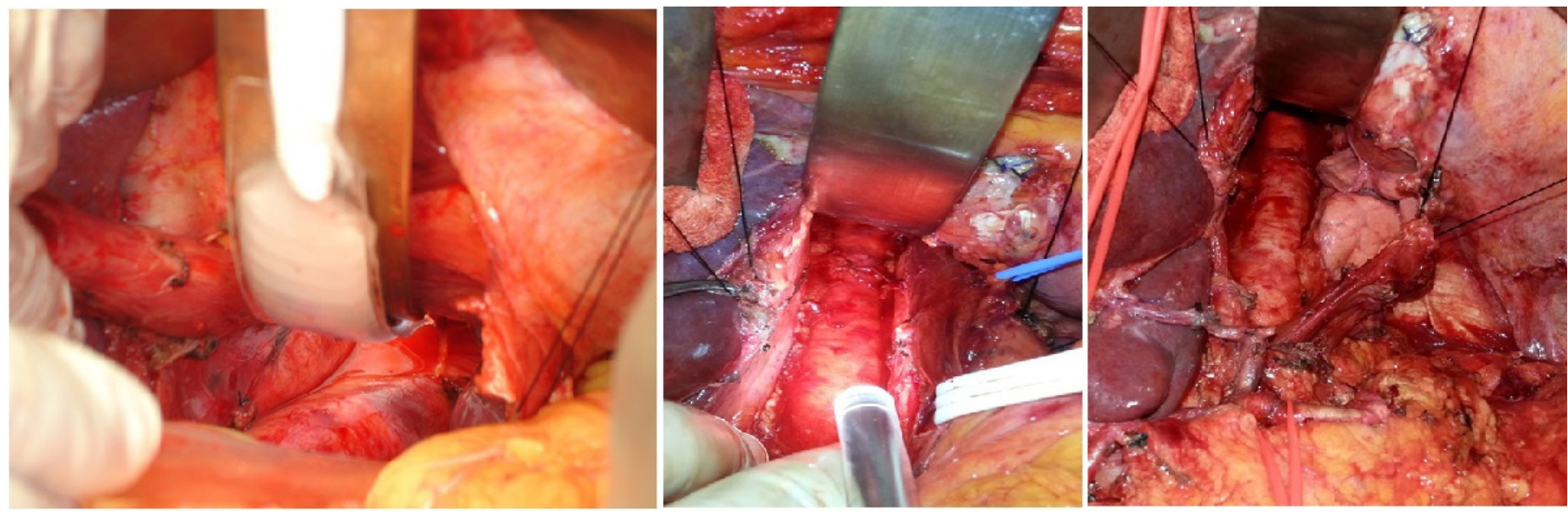

Figura 1 - Ressecção transhiatal 
da para a direita, com o pescoço em ligeira hiperextensão. A abordagem foi por via para-esterno-cleido-mastoideu esquerdo, com dimensão ajustada ao morfótipo do doente, para permitir o isolamento seguro do esófago, sem lesão dos eixos vasculares, dos nervos recorrentes ou da traqueia e para assegurar espaço adequado para a anastomose. Se não existia contra-indicação por razões oncológicas, o esófago foi mobilizado ligeiramente do tórax para o pescoço e seccionado com redundância, assegurando assim uma boa dimensão para garantir ausência de tensão na anastomose e implantação em continuidade linear com a plastia.

A anastomose esófago-gástrica ou esófago-cólica foi realizada como término-lateral manual, num plano de pontos separados, sutura reabsorvível. A sonda naso-gástrica (ou naso-cólica) foi passada para dentro da plastia antes de terminar a anastomose, para facilitar a manobra e garantir que não foram provocadas lesões. O esófago, a anastomose e a plastia foram recolocados junto à coluna, geralmente ao nível do opérculo. A cervicotomia foi encerrada após colocação de drenagem aspirativa.

O encerramento da laparotomia foi realizado por uma equipa, enquanto a outra procedeu à anastomose. $\mathrm{O}$ diafragma foi parcialmente encerrado, sem provocar estrangulamento do antro.

RTT - Esta abordagem pode fazer parte de uma estratégia cervico-tóraco-abdominal (três vias) ou tóraco-abdominal (duas vias).

$\mathrm{Na}$ abordagem por três vias, seguimos o procedimento semelhante ao praticado em Brigham. ${ }^{28} \mathrm{O}$ doente foi posicionado em decúbito lateral esquerdo, foi assegurada a exclusão pulmonar direita com controlo broncoscópico e a esofagectomia foi realizada por toracotomia direita $\left(5^{\circ}-6^{\circ}\right.$ espaço intercostal). Depois da mobilização do esófago e da dissecção ganglionar (estações ganglionares 1, 2, 3, 7, $9,11,16,19,20,106,107,108,109,110,111,112)^{27}$ adequada (Fig. 2), o canal torácico foi geralmente laqueado de maneira electiva.

Após colocação de um tubo de drenagem torácica siliconado, o tórax foi encerrado e o doente reposicionado como para a via transhiatal. Nestes casos, o tempo abdominal e o tempo cervical foram realizados em todos os doentes na posição de decúbito dorsal e a anastomose efectuada com o esófago cervical.

A operação de Ivor-Lewis, ${ }^{29}$ realizada apenas por duas vias, iniciou-se por laparotomia mediana, que foi encerrada após terminar o tempo abdominal de preparação da plastia e a dissecção ganglionar adequada. Os doentes foram seguidamente reposicionados para toracotomia, procedeu-se como referido anteriormente para a ressecção do esófago e territórios de drenagem linfáticos. A anastomose esófago-gástrica foi efectuada com agrafador circular introduzido pela porção proximal da plastia que viria a ser removida com a peça operatória. A preparação do tubo gástrico foi terminada no tórax para garantir a dimensão adequada da plastia.

Realçamos aqui apenas alguns detalhes da técnica da esofagectomia. A técnica de preparação do tubo gástrico foi recentemente publicada por nós. ${ }^{16} \mathrm{Na}$ construção do tubo gástrico e na sua mobilização foi tido em atenção: manipulação mínima do estômago, preservação cuidada da vascularização, manobra de Kocher para que o piloro seja mobilizável até ao hiato, piloromiotomia, anastomose esófago-gástrica término-lateral. A conservação da vascularização pelos vasos gastro-epiplóicos direitos e pilóricos, que asseguram a irrigação da plastia, são tempos cruciais e determinantes da viabilidade do tubo gástrico. Tendo a artéria coronária sido laqueada na origem, por exigência da dissecção ganglionar, a arcada vascular da pequena curvatura foi seccionada ao nível mais proximal possível, mas suficiente para permitir a remoção dos gânglios das estações 1, 2, 3 juntamente com as 7, 8, 9 e 11 e também para permitir o início da secção do estômago. 0 grande epiplon foi seccionado a 2-3 cm da arcada da grande curvatura, desde a origem dos vasos gastro-epiplóicos direitos até à origem da artéria gastro-epiplóica esquerda, junto à artéria esplénica. Para tal, os vasos curtos foram seccionados junto ao hilo do baço e procurou-se não interromper o indelével plexo venoso que se encontra no epiplon entre a
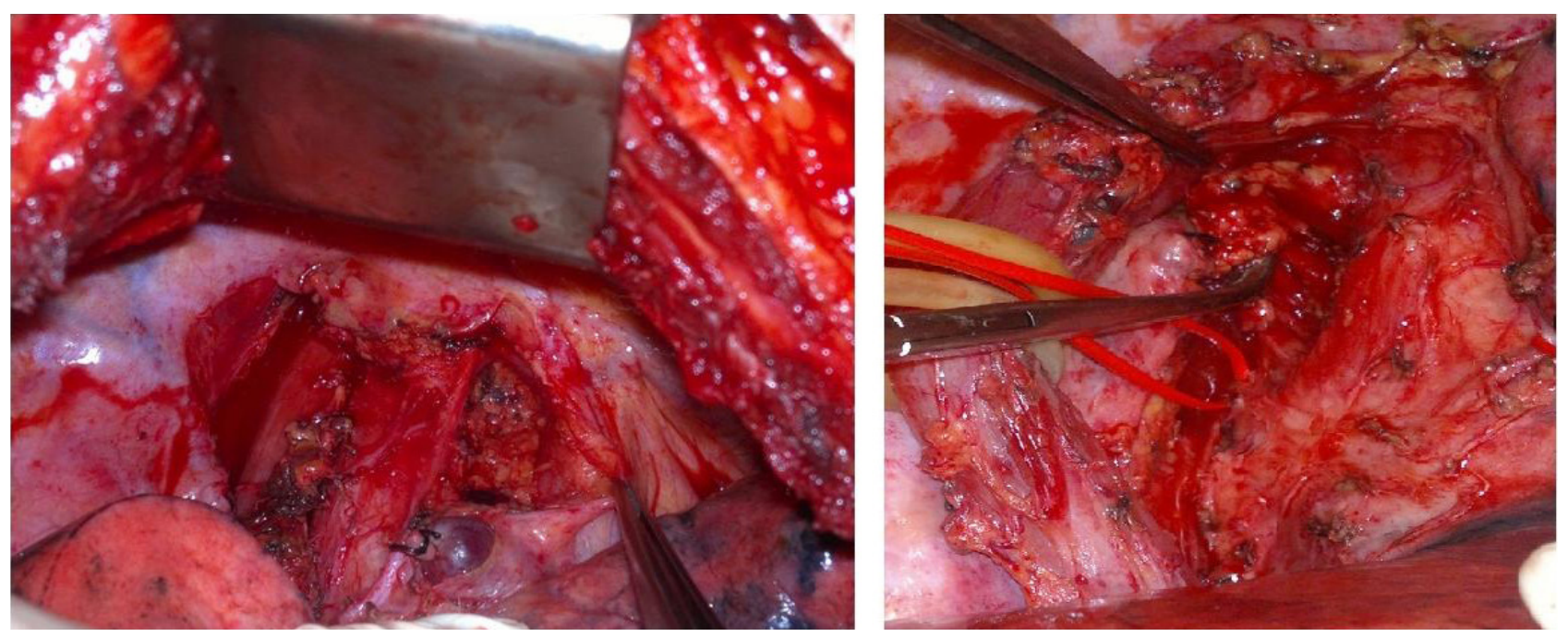

Figura 2 - Ressecção transtorácica. Aspecto do mediastino superior. 
porção esquerda da arcada gastro-epiplóca e os primeiros vasos curtos.

Nos casos em que a plastia foi realizada com o cólon, foi utilizada a técnica proposta por DeMeester ${ }^{30,31}$ para preparação de uma plastia isoperistáltica, irrigada pela artéria mesentérica inferior, com laqueação da artéria cólica média na origem (antes da bifurcação).

As equipas de anestesia e cirurgia devem ter uma interacção constante principalmente nos tempos críticos da dissecção transmediastínica e da transposição cervical, por ser necessário, por vezes, interromper momentaneamente o gesto para permitir a recuperação hemodinâmica. Aspectos críticos para a estabilidade homeostática: exclusão pulmonar aquando da toracotomia; monitorização das perdas de volume e sua reposição correcta, atempada e não exagerada para evitar o 'pulmão húmido'; cirurgia com perdas mínimas de sangue; minimização da lactacidose; monitorização da tensão arterial e das arritmias.

Foi feita terapêutica antibiótica profilática ou se indicado por algum evento infeccioso diagnosticado ou suspeito.

O pós-operatório imediato, foi realizado preferencialmente no Serviço de Medicina Intensiva ou no SO do nosso Serviço. A monitorização e o controlo homeostático 24/24 horas por Médicos Intensivistas residentes devem procurar a rápida extubação orotraqueal e, nas situações de necessidade de suporte ventilatório mais prolongado, assegurar o 'desmame' precoce da ventilação assistida. A atempada acção diagnóstica e terapêutica das alterações hemodinâmicas e metabólicas é essencial para a vitalidade das plastias. As aminas vasopressoras foram utilizadas judiciosamente, com titulação da posologia a intervalos curtos, para permitir a sua suspensão. A monitorização sistemática dos parâmetros inflamatórios foi assegurada. Na presença de derrame pleural pós-operatório equacionou-se a sua drenagem. A broncoscopia com intenção diagnóstica e terapêutica foi realizada muito liberalmente. A hemofiltração e a hemodiálise foram providenciadas sempre que tidas como necessárias. Foi feita profilaxia anti-trombótica com heparina de baixo peso molecular em todos os doentes, desde o dia um de pós-operatório.

No diagnóstico e tratamento das fístulas anastomóticas seguimos a classificação de Lerut $^{32}$ e os procedimentos que apresentámos recentemente. ${ }^{16}$

$\mathrm{Na}$ apresentação dos resultados recorreu-se a estatística descritiva e às curvas de Kaplan-Meier para cálculo da sobrevida actuarial.

\section{RESULTADOS}

- A necessidade de transfusões de sangue desde a véspera da intervenção, no intra-operatório e até às 48 horas de pós-operatório dos doentes submetidos a esofagectomia foi a seguinte: a) Zero UCE em 23 doentes; b) Uma UCE em seis doentes; c) Duas UCE em 14 doentes e d) Três UCE, ou mais, em sete doentes.

- Taxa de ressecabilidade: 96\% (50/52).

- Estadiamento patológico: regressão completa - 8 (RTT - 5, RTM - 3); lb - 3 (RTT - 0, RTM - 3); lla - 9 (RTT
- 6, RTM - 3); Ilb - 4 (RTT - 2, RTM - 2); Ila - 11 (RTT

- 5, RTM - 6); IIIb - 2 (RTT - 1, RTM - 1); IIlc - 10 (RTT

- 2, RTM - 8); IV - 1 (RTT - 0, RTM - 1); linfoma -1; não classificáveis -3 .

- Estádios III e IV - 26 (52\%).

- Envolvimento microscópico das margens distais em cinco doentes $(10 \%)$, pelo que a ressecção R0 foi conseguida em $90 \%$.

- Número de gânglios ressecados (média) - 16.

- A mediana do tempo de internamento foi 24 dias [9 -129].

- Complicações significativas em $37 \%$ dos casos: Fístulas anastomóticas - três doentes (duas clínicas de Grau II + uma radiológica de Grau I); Lesão recorrente - dois doentes; Lesão canal torácico - um doente; Derrame pleural - dois doentes; Pneumonia nosocomial - quatro doentes; ARDS - quatro doentes; Fístula bronco-pleural - um doente; Hemotórax com hemopericárdio - um doente; EAM - um doente; Aplasia medular - um doente; Seroma ferida cervical - um doente; Hemorragia gástrica controlada por endoscopia - um; Estase gástrica corrigida com procinéticos $-30 \%$.

- Não se verificou nenhuma necrose da plastia.

- Mortalidade às quatro semanas de $6 \%$ e hospitalar de $14 \%$.

A mortalidade por RTM foi três casos e RTT ocorreu em quatro doentes. Destes sete doentes, cinco tinham sido incluídos no esquema de terapêutica neo-adjuvante.

- Causas de morte até às oito semanas: uma fístula broncopleural + pneumonia nosocomial; uma aplasia medular aguda; um motivo desconhecido. Mortalidade hospitalar: dois ARDS (pneumonite intersticial); um EAM; um motivo desconhecido.

- Todos os doentes tiveram alta sem limitações disfágicas, com capacidade alimentar autónoma, com indicação para aumento progressivo do volume e diversificação da ingestão de alimentos, segundo aconselhamento com o Nutricionista do Serviço.

- Onze doentes apresentaram queixas de disfagia no pós-operatório. Quando o controlo radiológico demonstrou estenose da anastomose, os doentes foram submetidos a dilatações endoscópicas. Geralmente foi necessária mais que uma dilatação e estas ocorreram quase sempre no primeiro ano após a cirurgia.

- Sobrevida mediana de 18 [3-80] meses. Durante este período não foi necessária qualquer intervenção operatória relacionada com o funcionamento do tubo gástrico.

- Curvas de sobrevida (Kaplan-Meier): 47\% sobrevida aos dois anos e 19\% aos cinco anos (Fig. 3).

Não foram encontradas diferenças significativas na sobrevivência dos doentes operados por via RTM em relação aos operados por RTT: $p>0,05$ (Fig. 4).

Não se encontraram diferenças significativas quanto à sobrevivência dos doentes com adenocarcinoma vs carcinoma pavimento celular $(p>0,05)$, nem nos doentes com remissão completa $(p>0,05)$. 


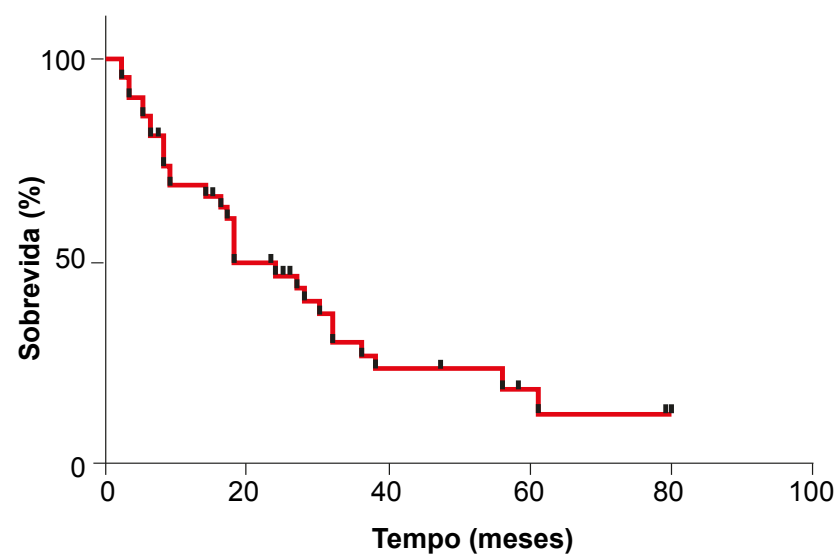

Figura 3 - Sobrevida Global

\section{DISCUSSÃO}

A agressividade biológica e oncológica dos tumores do esófago é responsável pelos resultados menos favoráveis geralmente publicados. Este pressuposto está na origem das diversas vias de investigação para minimizar os riscos das terapêuticas, melhorar a qualidade de vida dos doentes e aumentar a sobrevida. Nas últimas décadas a ressecabilidade dos tumores aumentou substancialmente, a morbi-mortalidade dos procedimentos cirúrgicos e/ou combinados tem vindo a melhorar, mas na sobrevida à distância e nas recidivas locais não têm sido conseguidos progressos equivalentes. ${ }^{2,10,12,13,23}$

Actualmente a cirurgia continua a ter um papel bem definido nos principais protocolos de estratégia do cancro do esófago, nas variedades epidermóide e adenocarcinoma. ${ }^{5-9 ; 17}$

Considerações de raiz oncológica, ligadas à radicalidade dos procedimentos, não têm sido exemplarmente demonstradas em ensaios bem desenhados e conduzidos por grupos experientes, nem as metanálises elaboradas foram determinantes na selecção da extensão e radicalidade operatórias. ${ }^{10-14}$

A questão da determinação da equivalência, ou da superioridade, da ressecção transmediastínica (RTM) em comparação com abordagem transtorácica (RTT), que permite maior radicalidade na ressecção do mediastino superior, não está resolvida. A ressecção transmediastínica e a transtorácica têm atualmente taxas mortalidade hospitalar entre $1 \%$ - 20\% ${ }^{32}$ (tendencialmente maior para as RTT com ressecções mais alargadas) e, na generalidade das melhores séries, as sobrevivências são equivalentes entre ambas, de cerca de $25 \%$ aos cinco anos. , $^{1,12-15,34-36}$

Os prós e contras de cada alternativa encontram-se difusamente explicitados nas experiências individuais e revisões sistemáticas que temos vindo a referir.

$\mathrm{Na}$ ausência de superioridade oncológica evidenciada por qualquer uma das abordagens para esofagectomia (RTM ou RTT), ,10-15 propusemos uma estratégia de abordagem orientada pelo doente e pela localização do tumor. Assim, os tumores localizados num plano inferior à carina

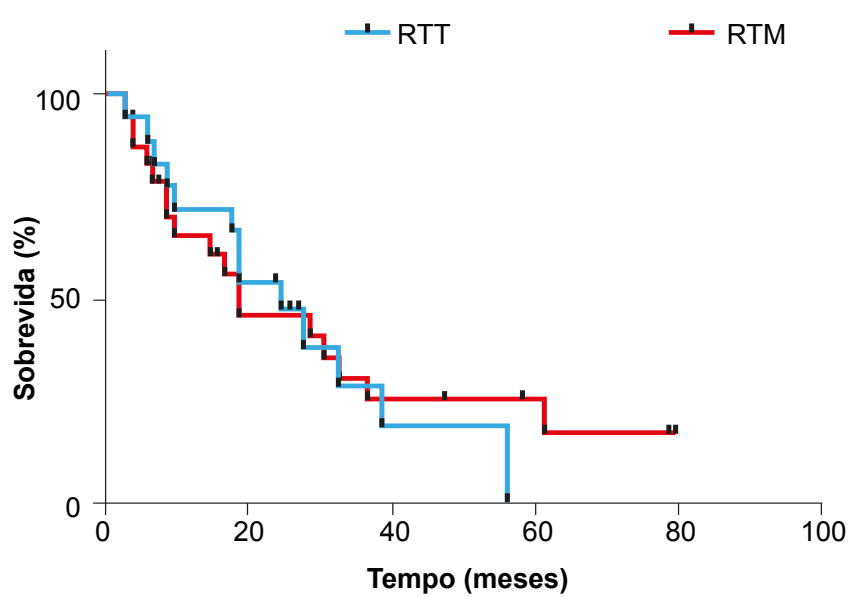

Figura 4 - Sobrevida RTT vs. RTM

foram operados por RTM, com frenotomia extensa, iluminação dirigida e visualização direta do tumor, dos tecidos envolventes e das cadeias de drenagem linfática infra-carinais e mediastínicas inferiores ${ }^{27}$ que puderam ser ressecados com segurança. Os tumores cervicais podem também ser abordados por RTM, mas raramente têm indicação operatória. Os tumores torácicos altos foram operados por RTT com dissecção ganglionar já referida. ${ }^{27}$

A padronização da estratégia de abordagem cirúrgica, para tratamento dos tumores do esófago, contribuiu para os resultados que obtivemos nos últimos 50 doentes que tratámos, em ambiente multidisciplinar. Para tal, um dos autores participou em todas as operações como um dos cirurgiões sénior das equipas do Serviço envolvidas. Nos centros de médio volume de esofagectomias, o case-mix do Serviço, a sua diferenciação em oncologia digestiva, resultante do aumento de referenciação e treino dos cirurgiões envolvidos, têm sido apontados como os factores que condicionam a obtenção de resultados equivalentes a outros centros com maior volume desta patologia. . $^{3,37,38}$

Os nossos resultados estão a par com os encontrados por outros grupos, com sobrevida global mediana de 18 meses, sem diferenças significativas $(p>0,05)$ para as duas abordagens (RTM e RTT). A sobrevida de $47 \%$ aos dois anos com uma perda acentuada aos cinco anos (19\%) é semelhante descrita na literatura. . $11,12-15,34,35^{2}$

Alguns trabalhos prospectivos recentes ${ }^{39-41}$ apresentam sobrevidas semelhantes para as duas técnicas, mas com tempo livre de doença maior para a RTT.

A técnica operatória, num processo cirúrgico tão complexo como a esofagectomia, pode condicionar os resultados imediatos e à distância. Os resultados imediatos ponderam-se essencialmente pela ressecabilidade, a morbilidade e a mortalidade. Estes resultados influenciam de maneira significativa os resultados à distância da esofagectomia por cancro (a sobrevivência).

A reduzida necessidade de transfusões de sangue no peri-operatório (zero unidades em $46 \%$ dos casos) está relacionada com a opção estratégica seguida, quanto à via de abordagem por RTM vs RTT, que permitiu um acesso 
visual e adequado dos territórios com maior exigência técnica, ao treino da equipa e aos recursos de iluminação e selagem de vasos, disponíveis. Sobretudo na RTM, a frenotomia bem desenvolvida e estes recursos tecnológicos tornaram uma intervenção de execução geralmente baseada na sensibilidade táctil, num processo que hoje realizamos quase na totalidade sob controlo visual e acesso direto. Na RTM utilizámos inicialmente uma fonte de iluminação que transmitia luz por fitas auto-colantes aos instrumentos cirúrgicos, geralmente a um afastador. Mais recentemente temos utilizado o videoscópio.

O número de gânglios ressecados (média) 16 e as margens negativas em 45 doentes (90\%) R0, estão de acordo com o geralmente descrito. ${ }^{42}$

Analisámos a nossa experiência com a utilização do tubo gástrico na reconstrução digestiva após esofagectomia por cancro gástrico. Realizámos preferencialmente a anastomose esófago-gástrica cervical com excelentes resultados, ${ }^{16}$ quando cotejados com a literatura recente..$^{15,17,33}$ A taxa de complicações relacionadas com o enxerto situou-se numa faixa muito baixa. Observámos fístulas anastomóticas ${ }^{32}$ em três doentes (duas clínicas de Grau II + uma radiológica de Grau I), uma hemorragia gástrica controlada por endoscopia, e estase gástrica corrigida com procinéticos em cerca de $30 \%$. Os valores apontados para as fístulas desta anastomose variam entre 9,6-16\%. ${ }^{2,10,11,33}$ Não se verificou nenhuma necrose da plastia ao contrário do descrito por outros. . $^{33,43,44}$

A taxa de estenoses da anastomose cervical, $22 \%$, com necessidade de dilatações endoscópicas é um inferior à de outras séries de referência. ${ }^{15}$

Os dados de uma metanálise recente,${ }^{15}$ sobre a problemática da anastomose cervical vs torácica, não permitiram determinar uma evidência forte a favor de qualquer das opções. Estes dados mais não trazem que reforçar os resultados de outros trabalhos ${ }^{2,10-14,23}$ que apontam para uma taxa ligeiramente superior de fístulas e estenoses quando a anastomose é realizada no pescoço. Mas a 'benignidade' destas complicações, comparada com as mesmas quando ocorrem no tórax, é para nós um factor de ponderação fortemente positiva a favor da anastomose cervical. Quando utilizamos o mediastino posterior e posicionamos bem o esófago e o tubo, sem tensão e em continuidade vertical, parece-nos que esta é realmente uma boa alternativa. No entanto, não podemos deixar de referir o maior risco de complicações pulmonares que pode estar associado à anastomose cervical. ${ }^{10,12,15,16}$

A morbi-mortalidade da esofagectomia, em doentes com cancro do esófago, melhorou muito nas últimas décadas, mantendo-se, no entanto com um peso não negligenciável (morbilidade: $38-60 \%$; mortalidade 30 dias: $0-22 \%) .2,23,33,34$

Os nossos resultados, complicações significativas em $37 \%$ dos casos, com mortalidade às quatro semanas de $6 \%$, mas mortalidade hospitalar de $14 \%$, são a tradução dessa realidade.

Muitos autores têm procurado algoritmos de decisão de risco operatório com a intenção de reduzir a morbi-mortalidade com base numa mais apurada seleção de doentes, leia-se, reduzir os critérios de admissibilidade à cirurgia. ${ }^{24,25,34,45-52} \mathrm{O}$ fit for surgery pretende-se que passe cada vez mais por cálculos de probabilidade e menos pela impressão clínica. Os principais órgãos alvo de complicações foram o pulmão e o coração. Não se encontrou ainda o algoritmo padrão e nesta série de doentes procedemos a avaliações funcionais semelhantes para nos ajudarem a tomar a decisão individual, dificilmente dedutível dos nomogramas. ${ }^{51,53}$

A terapêutica neoadjuvante (quimioterapia, radioterapia ou sobretudo a quimio-radioterapia) pode aumentar a ressecabilidade, contribuir para melhorar a sobrevida dos doentes e/ou o período livre de doença, sem aumentar significativamente a morbi-mortalidade, quando a radioterapia é focada para reduzir a exposição pulmonar. ${ }^{6,7,23,54,55}$

A elevada taxa de ressecabilidade (96\%), que conseguimos nesta série consecutiva de doentes que propusemos para esofagectomia, deveu-se a uma criteriosa avaliação pré-operatória e à inclusão de doentes em programa de terapêutica neo-adjuvante.

A quimio-radioterapia foi prescrita em doentes clinicamente $\mathrm{N}+$ e nos T3-4 para reduzir a massa tumoral e permitiu a boa taxa de ressecção nos doentes selecionados para cirurgia.

A inclusão em esquemas de terapêutica neo-adjuvante aumentou nos últimos anos, por uma melhor integração multidisciplinar no nosso hospital, embora fosse essa a nossa perspectiva desde há anos. Em 1983 apresentámos os resultados preliminares da metodologia da avaliação da quimioterapia pré-operatória no carcinoma do esófago [Costa P, Costa B, Silveira JC, Camilo E, Guerreiro D, Veiga Fernandes F. Metodologia da avaliação da quimioterapia pré-operatória no carcinoma do esófago. (dados pessoais, III Congresso Nacional de Cirurgia, 1983).]. Os objectivos, por trás das palavras da época, eram diminuir a massa e a carga tumorais, aumentar a ressecabilidade, ganhar tempo para conseguir uma optimização biológica, melhorar a avaliação do risco/selecção dos doentes, e, como consequência, diminuir a mortalidade. Estes desideratos continuam a fundamentar a terapêutica que actualmente designamos por neo-adjuvante. . $, 6,7,22,23$

Não encontrámos diferenças significativas quanto à sobrevivência dos doentes com adenocarcinoma vs carcinoma pavimento celular $(p>0,05)$, nem nos doentes com remissão completa $(p>0,05)$.

O significado real da regressão tumoral completa, referida em valores entre $20-40 \%$, ainda não está determinado, quanto à sua influência na sobrevida global e livre de doença, no desenvolvimento de recorrência local e à distância [Tato Costa J, Costa L, Casimiro S. Effects of chemotherapy and radiotherapy in rectal cancer: significance of different cellular outcomes in tumour behaviour. (dados pessoais Projecto de Tese de Doutoramento - IMM/FMUL, em execução)]. A presença de células tumorais senescentes, que perderam a capacidade de divisão, mesmo quando esti- 
muladas por mitogénios tem sido descrita nas remissões completas. Estas células, ficam metabólica e sinteticamente activas (PDGF, VEGF, EGF, TGF- $\alpha$, etc.), podendo vir a cooperar no desenvolvimento de recidivas locais. Estas recidivas têm sido abordadas por salvage surgery com bons resultados.

\section{CONCLUSÃO}

Não tendo sido demonstrada vantagem oncológica substantiva para a RTT ou a RTM, basear a opção operatória na localização do tumor permitiu-nos com segurança e eficácia, planear e executar as ressecções esofágicas desta série de carcinomas.

As curvas de sobrevida foram sobreponíveis para a RTT e RTM e os valores encontrados foram bastante favo- ráveis para uma população em que $26,5 \%$ dos casos foram estadiados como III/IV.

A quimio-radioterapia contribuiu para aumentar a ressecabilidade dos tumores e em $42 \%$ dos casos submetidos induziu uma remissão completa.

\section{OBSERVAÇÕES}

Trabalho parcialmente apresentado no XXXIII Congresso Nacional de Cirurgia, 2013.

\section{CONFLITOS DE INTERESSE}

Sem conflitos de interesse.

\section{FONTES DE FINANCIAMENTO}

Sem suporte financeiro.

\section{REFERÊNCIAS}

1. Pennathur A, Zhang J, Chen H, Luketich J. The "Best operation" for esophageal cancer? Ann Thorac Surg. 2010;89:S2163-7.

2. Lagarde SM, Vrouenraets BC, Stassen LP, Van Lanschot JJ. Evidencebased surgical treatment of esophageal cancer: overview of high-quality studies. Ann Thorac Surg. 2010;89:1319-26.

3. Dikken JL, Wouters MW, Lemmens VE, Putter H, Van der Geest LG, Verheij M, et al. Influence of hospital type on outcomes after oesophageal and gastric cancer surgery. Br J Surg. 2012;99:954-63.

4. Boddy AP, Williamson JML, Vipond MN. The effect of centralization on the outcomes of oesophagogastric surgery - a fifteen years audit. Int J Surg. 2012;10:360-3

5. Worni M, Martin J, Gloor B, Pietrobon R, D'Amico TA, Akushevich I, et al. Does surgery improve outcomes for esophageal squamous cell carcinoma? An analysis using the surveillance epidemiology and end results registry from 1998 to 2008. J Am Coll Surg. 2012;215:643-51.

6. Werutsky G, Ducreux M, Lutz M, Mauer M, Van Clutsem E, Ruers T, et al. The EORTC gastrointestinal tract cancer group: 50 years of research contributing to improved gastrointestinal cancer management. EJC supplements IO. 2012;1:51-7.

7. Gebski V, Burmeister B, Smithers BM, Foo K, Zalcberg J, Simes J. Survival benefits from neoadjuvant chemo radiotherapy or chemotherapy in oesophagal carcinoma: a meta-analysis. Lancet Oncol. 2007;8:22634.

8. Abrams JA, Buono DL, Strauss J, McBride RB, Hershman DL, Neugut Al. Esophagectomy compared with chemoradiation for early stage esophageal cancer in the eldery. Cancer. 2009;115:4924-33.

9. NCCN Guidelines Version 1. 2013 Sub-Committees Esophageal and Esophagogastric Junction Cancers; [Consultado 2013 Abr 7]. Disponível em: http://www.nccn.org/professionals/physician_gls/f_guidelines. asp\#site.

10. Hulscher JB, Tijssen JG, Obertop H, van Lanschot JJ. Transthoracic versus transhiatal resection for carcinoma of the esophagus: a metaanalysis. Ann Thorac Surg. 2001;72:306-13

11. Orringer MB. Transhiatal esophagectomy without thoracotomy. In: Cohn LH, Patterson A editors. Operative Techniques in Thoracic and Cardiovascular Surgery. Philadelphia: Elsevier Spring; 2005. p. 63-83.

12. Yang K, Chen H, Chen XZ, Lu QJ, Pan L, Liu J, et al. Transthoracic resection versus non-transthoracic resection for gastroesophageal junction cancer: a meta-analysis. Plos one. 2007;6:e37698.

13. Chang AC, Ji H, Birkmeyer NJ, Orringer MB, Birkmeyer JD. Outcomes after transhiatal and transthoracic esophagectomy for cancer. Ann Thorac Surg. 2008;85:424-9.

14. Barreto JC, Posner MC. Transhiatal versus transthoracic esophagectomy for esophageal cancer. World J Gastroenterol. 2010;16:3804-10.

15. Bierre SS, Maas KW, Cuesta MA, van der Peet DL. Cervical or thoracic anastomosis after esophagectomy for cancer: a systematic review and meta-analysis. Dig Surg. 2011;28:29-35.

16. Costa $P$, Esteves $R$, Lages $P$, Ferreira F. Transposição cervical de tubo gástrico na reconstrução digestiva após esofagectomia por cancro do esófago - detalhes técnicos. Rev Port Cirurgia. 2013;25:9-21.

17. Oezcelik A, Kaiser GM, Niebel W, Sleyman C, Treckmann JW, Sotiropulos GC, et al. Ten-year survival of esophageal cancer after an en-bloc esophagectomy. J Surg Oncol. 2012;105:284-7.

18. Wong RK, Malthaner RA, Zuraw L, Rumble RB. Combined modality radiotherapy and chemotherapy in nonsurgical management of localized carcinoma of the esophagus: a practice guideline. Int J Radiat Oncol Biol Phys. 2003;55:930-42

19. Yamada S, Nemoto K, Ariga H, Jingu K. Radiotherapy for esophageal cancer in Japan. Esophagus. 2012;9:66-74.

20. Atsumi K, Shioyama Y, Arimura H, Terashima K, Matsuki T, Ohga S, et al. Esophageal stenosis associated with tumor regression in radiotherapy for esophageal cancer: frequency and prediction. Int J Radiation Oncol Biol Phys. 2012;85:1973-80.

21. Nishimura $Y$, Koike R, Ogawa K, Sasamoto R, Murakami $Y$, Itoh $Y$, et al. Clinical practice and outcome of radiotherapy for esophageal cancer between 1999 and 2003: the Japanese Radiation Oncology Study Group (JROSG) survey. Int J Clin Oncol. 2012;17:48-54.

22. $\mathrm{Xu} Y, \mathrm{Yu} X$, Chen $\mathrm{Q}$, Mao W. Neoadjuvant versus adjuvant treatment: which one is better for ressectable esophageal squamous cell carcinoma? World J Surg Oncol. 2012;10:173-80.

23. Merrit RE, Whyte RI, D'Arcy NT, Hoang CD, Shrager JB. Morbidity and mortality after esophagectomy following neo adjuvant chemoradiation. Ann Thorac Surg. 2011;92:2034-40.

24. Hashimi S, Smith M. Medical evaluation of patients preparing for an esophagectomy. Surg Clin N Am. 2012;92:1127-33

25. Grotenhuis BA, Wijnhoven BP, Grune F, Van Bommel J, Tilanus HW, Van Lanschot JJ. Preoperative risk assessment and prevention of complications in patients with esophageal cancer. J Surg Oncol. 2010;101:270-8.

26. Pinotti HV. Acesso ao esôfago torácico por transecção mediana do diafragma. São Paulo: Atheneu; 1999.

27. Japan Esophageal Society. Japanese Classification of Esophageal Cancer, Tenth Edition: Parts II and III. Esophagus. 2009;6:71-94.

28. Swanson SJ, Batirel HF; Bueno R. Transthoracic esophagectomy with radical mediastinal and abdominal lymph node dissection and cervical esophagogastrostomy for esophageal carcinoma. Ann Thorac Surg 2001;72:1918-25.

29. Lewis I. The surgical treatment of carcinoma of the esophagus, with special reference to a new operation for growths of the middle third. $\mathrm{Br}$ J Surg. 1946;34:18-31.

30. DeMeester TR, Johansson KE, Franze J, Eypasch E, Lu CT, McGill JE, et al. Indications, surgical technique, and long-term functional results of colon interposition or bypass. Ann Surg. 1988;208:460-74.

31. DeMeester TR. Esophageal replacement with colon interposition. Oper Tech Cardiac Thorac Surg. 1997;2:73-86.

32. Lerut $T$, Coosemans $W$, De Leyn $P$, Nafteux $P$, van Raemdonck $D$. Anastomotic complications after esophagectomy. Dig Surg. 2002;19:92-

33. Raymond D. Complications of esophagectomy. Surg Clin N Am. 2012;92:1299-313.

34. Gockel I, Exner C, Junginger T. Morbidity and mortality after esophagectomy for esophageal carcinoma: a risk analysis. World J Surg Oncol. 2005;3:37-44.

35. Tachibana M, Kinugasa S, Yoshimura H, Shibakita M, Tonomoto Y, Dhar 
DK, et al. En-bloc esophagectomy for esophageal cancer. Am J Surg. 2004;188:254-60.

36. Rentz J, Bull D, Harpole D, Bailey S, Neumayer L, Pappas T, et al. Transthoracic versus transhiatalesophagectomy: a prospective study of 945 patients. J Thorac Cardiovasc Surg. 2003;125:1114-20.

37. Rouvelas I, Lindblad M, Zeng W, Viklund P, Ye W, Lagerland J. Impact of hospital volume on long-term survival after esophageal cancer surgery. Arch Surg. 2007;142:113-7.

38. Kozower BD, Stukenborg GJ. Hospital esophageal cancer resection volume does not predict patient mortality risk. Ann Thorac Surg. 2012;93:1690-8.

39. Stiles BM, Altorki NK. Traditional techniques of esophagectomy. Surg Clin N Am. 2012;92:1249-63.

40. Hulscher JB, Sandick JW, De Boer AG; Wijnhoven BP, Tijssen JG, Fockens JG, et al. Extended trahansthoracic resection compared with limited transhiatal resection for adenocarcinoma of the esophagus. $\mathrm{N}$ Engl J Med. 2002;347:1662-9.

41. Schröder W; Schneider PM; Hölscher AH. Transthoracic en-bloc esophagectomy. The Chinese-German J Clin Oncol. 2004;3:229-32.

42. Herrera JL. Extent of lymphadenectomy in esophageal cancer: how many lymph nodes is enough? Ann Surg Oncol. 2010;17:676-8.

43. Schweigert M, Solymosi N, Dubecz A, Stadlhuber RJ, Muschweck $\mathrm{H}$, Ofner D, et al. Endoscopic stent insertion for anastomotic leakage following oesophagectomy. Ann R Coll Surg Engl. 2013;95:43-7.

44. Briel JW, Tamhankar AP, Hagen JA, DeMeester SR, Johansson J, Choustoulakis E, et al. Prevalence and risk factors for ischemia, leak, and stricture of esophageal anastomosis: gastric pull-up versus colon interposition. J Am Coll Surg. 2004;198:536-42.

45. Bailey SH, Harpole DH, Rentz JJ, Neumayer LA, Pappas TN, Daley J, et al. Outcomes after esophagectomy: a ten-year prospective cohort. Ann Thorac Surg. 2003;75:217-22.

46. Law S, Wong KH, Kwok KF, Chu KM, Wong J. Predictive factors for postoperative pulmonary complications and mortality after esophagectomy for cancer. Ann Surg. 2004;240:791-800.

47. Ferguson MK, Celauro AD, Prachand V. Prediction of major pulmonary complications after esophagectomy. Ann Thorac Surg. 2011;91:1494501.

48. Bakhos CT, Fabian T, Oyasiji TO, Gautam S, Gangadharan SP, Kent MS, et al. Impact of surgical technique on pulmonary morbidity after esophagectomy. Ann Thorac Surg. 2012;93:221-7.

49. Togo S, Ligang L, Wei X, Tiechang $P$, Chen T, Ouattara MA, et al Complications and mortality after esophagectomy for esophageal carcinoma: risk factor analysis in a series of 378 patients. Chir Thorac Cardio-Vasc. 2010;14:25-8.

50. Bosch DJ, Pultrum BB, Bock GH, Oosterhuis JK, Rodgers MG, Plukker JT. Comparison of different risk-adjustment models in assessing shortterm surgical outcome after transthoracic esophagectomy in patients with esophageal cancer. Am J Surg. 2011;202:303-9.

51. Moyes LH, McCaffer CJ, Carter RC, Fullarton GM, Mackay CK, Forshaw MJ. Cardiopulmonary exercise testing as a predictive of complications in oesophagogastric cancer surgery. Ann R Coll Surg Engl. 2013;95:12530.

52. Hashimi S, Smith M. Medical evaluation of patients preparing for an esophagectomy. Surg Clin N Am. 2012;92:1127-33.

53. Grotenhuis BA, Van Hagen P, Reitsma JB, Lagarde SM, Wijnhoven BP van Berge Henegouwen, et al. Validation of a normogram predicting complications after esophagectomy for cancer. Ann Thorac Surg. 2010;90:920-6.

54. Reynolds JV, Ravi N, Hollywood D, Kennedy MJ, Rowley S, et al Neo adjuvant chemoradiation may increase the risk of respiratory complications and sepsis after transthoracic esophagectomy. J Thorac Cardiovasc Surg. 2006;132:549-55.

55. Reim D, Gertier R, Novotny A, Becker K, zum Buschenfelde CM, Ebert $M$, et al. Adenocarcinomas of the esophagogastric junction are more likely to respond to preoperative chemotherapy than distal gastric cancer. Ann Surg Oncol. 2012;19:2108-18. 


\section{Cancro do Esófago: Estratégia de Abordagem Cirúrgica}

Acta Med Port 2014:27:593-600

Publicado pela Acta Médica Portuguesa, a Revista Científica da Ordem dos Médicos

Av. Almirante Gago Coutinho, 151

1749-084 Lisboa, Portugal.

Tel: +351218428215

E-mail: submissao@actamedicaportuguesa.com

www.actamedicaportuguesa.com

ISSN:0870-399X | e-ISSN: 1646-0758

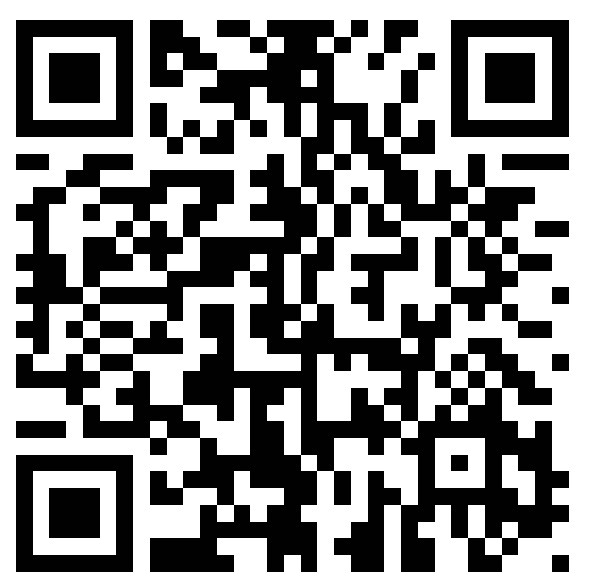

